\title{
Editorial: Recent Advances and Applications of Hybrid Simulation
}

\author{
Wei Song ${ }^{1 *}$, Chia-Ming Chang ${ }^{2}$ and Vasilis K. Dertimanis ${ }^{3}$ \\ ${ }^{1}$ Department of Civil, Construction and Environmental Engineering, University of Alabama, Tuscaloosa, AL, United States, \\ ${ }^{2}$ Department of Civil Engineering, National Taiwan University, Taipei, Taiwan, ${ }^{3}$ ETH Zurich, Zurich, Switzerland
}

Keywords: hybrid simulation, real-time hybrid simulation, compensation algorithm, stability analysis, uncertainty

\section{Editorial on the Research Topic}

\section{Recent Advances and Applications of Hybrid Simulation}

The smooth operation of our built environment and modern society heavily rely on the design and construction of large structural systems. Examples include buildings, bridges, and wind turbines. These systems, which serve important daily functions, such as human habitat, transportation, power generation and transmission, often face complex and demanding operation conditions throughout their service life. To ensure the structural integrity and operation safety of these systems and improve their future design, it is important to reveal their complex behavior in various operation environments, especially doing so through experimentations that can replicate realistic working conditions.

Conventional structural testing platforms alone, such as shake table and wind tunnel, may have difficulties in accommodating the entirety of such large- or full-scale structural systems due to limitations in laboratory space, equipment capacity, or fabrication cost. Hybrid simulation provides an attractive alternative for testing large and complex structural systems. This testing method allows physical testing of only the part of the system that is of interest, usually exhibiting complex behavior or yet to be fully understood, while the rest of the system is simulated simultaneously using numerical models with good accuracy and confidence. Such a substructure technique not only provides cost-savings and insights into detailed local behavior of the physical subsystem, but also offers better understanding of the entire complex structural systems, particularly of those systems with multiple components and complex interactions.

A successful hybrid simulation depends on several key factors, including a well-conceived experimental subsystem closely representing realistic boundary conditions, a high-fidelity yet efficient numerical subsystem, a robust and reliable transfer system interfacing numerical and physical subsystems, and a partitioning configuration promoting stability and accuracy in realtime hybrid simulation. As researchers are pushing the boundaries of new capabilities and better enabling tools for hybrid simulation, its benefit on characterizing complex structural behavior is driving the growth of hybrid simulation in broader engineering fields. This Research Topic on Recent Advances and Applications of Hybrid Simulation aims to deepen the knowledge of novel theories and enabling techniques for hybrid simulation and to broaden the spectrum of hybrid simulation applications and studies. Research works collected through this topic closely represent the advancement in the above fronts and are summarized in this editorial.

This Research Topic opens with new applications of hybrid simulation on revealing the structural behavior of complex systems. In Harris and Christenson, a real-time hybrid simulation (RTHS) campaign is developed to capture the uncertainties in force profiles of moat wall impacts and to analyze the complex interactions between impacts and the dynamics of base-isolated structures during earthquake excitations. It is shown that the use of hybrid simulation fully captures the force-deflection behavior of the moat wall and the uncertainties associated with impact events, 
and illustrates the importance of designing for potential nonlinear behavior within the moat wall during impact events. In another application involves building structure, Mukai et al. conduct a RTHS to study the seismic response of a single-story reinforced concrete $(\mathrm{RC})$ frame building with an active mass damper (AMD). In this study, most parts of the RC frame are simulated as a numerical model, while a single column of the first story and a controllable AMD are physically tested as experimental subsystems. To improve the online numerical model based on the measured force responses of the RC column specimen, a high-pass filter (HPF) is applied for a force correction by utilizing its phase-lead property. The RTHS test results demonstrate the control performance of the AMD on increasing the damping of the target RC frame. Hybrid simulation has also been applied to study bridge behavior. In Yang et al., hybrid simulation techniques are used to study the seismic behavior of steel girder bridge supported on high-damping rubber (HDR) bearings. In the hybrid simulation tests, HDR bearings are physically tested, while other structural components including the bridge structure are numerically simulated. The results demonstrate that hybrid simulation is a reliable means to study the seismic performance of HDR bearings.

A hybrid simulation platform is a complex dynamic system by itself. Without a proper control strategy, the transfer system interfacing numerical and physical subsystems will experience amplitude variations and phase delays, cause possible instability of hybrid simulation and eventually lead to experimental failure. This effect is more profound in the case of RTHS where rate dependent behavior is dominant. Therefore, control algorithms that can ensure tracking performance of the transfer system are an essential enabling tool for RTHS. Tsokanas et al. propose a novel transfer system controller for time delay compensation. It consists of a robust model predictive control (MPC) along with a polynomial extrapolation algorithm and a Kalman filter. The tracking performance and robustness of the proposed controller is then verified by two virtual RTHS parametric case studies. The obtained results illustrate that the proposed tracking controller can guarantee very small time delays and tracking errors under uncertainties that may be present in RTHS. In Simpson et al., a data-driven control strategy is developed for RTHS. Unlike conventional controllers, where the control plant (transfer system and experimental subsystem) is modeled by a physics-based analytical model, the proposed approach relies exclusively on data processing for tuning an adaptive inverse controller for RTHS. The efficacy and robustness of the proposed control strategy is assessed via a virtual RTHS study. The obtained results indicate that the proposed approach may form a competitive alternative to conventional controllers in RTHS applications.

For a successful hybrid simulation, it is crucial to analyze the system stability of overall hybrid simulation platform to avoid potential damage to specimen or equipment. Gálmez and Fermandois develop a stability indicator that can be evaluated online during RTHS test. Derived from energy analysis, this indicator allows the detection of unstable behavior before the system reaches large displacements that can damage the experimental subsystem or laboratory equipment. Its effectiveness is demonstrated through a virtual RTHS study with linear system assumption and different compensation strategies. In Chen et al., a stability analysis is conducted for a RTHS for building mass damper (BMD) systems. In the RTHS design, the experimental subsystem includes the control layer and the superstructure of the BMD system installed on a shake table while the substructure is simulated as numerical subsystem. Through a stability analysis, the stability margin can be obtained as an allowable mass ratio of the experimental subsystem to the entire BMD system. This stability margin is then experimentally verified by a RTHS of a simplified BMD system. This study also represents an example of shake table substructure testing (STST), a technique combining hybrid simulation and shake table testing. Tian et al. provides a comprehensive review on STST based on its development in the past two decades, including hybrid simulation frameworks, time integration algorithms, delay compensation methods, shake table and actuator control schemes and boundary force measurement methods. The key techniques and challenges for future STST studies and applications are also identified and presented in this review.

By leveraging advanced modeling techniques and system analysis tools, new developments on its frameworks are empowering hybrid simulation with capabilities of tackling more complex structural systems and more efficient implementation. During hybrid simulation, response time history measured from an experimental subsystem can be utilized to identify the model associated with the tested specimen. Ou et al. present a detailed investigation on model fidelity improvement using hybrid simulation with model updating. This study is focused on both local and global assessment of the updating results by comparing it to conventional simulation and shake table testing. Using seismic response obtained from a steel building frame under shake table testing, this updating approach is successfully implemented to update both a Bouc-Wen model and a bilinear steel constitutive finite element model. In Maghareh et al., a modular framework is developed to enable a safe, confident, and effective RTHS application with challenging and realistic experiments. This framework, currently in its first phase, serves as a foundation for extending RTHS application to blackbox RTHS experiments. The building blocks of the framework are demonstrated and validated using numerical studies on a virtual RTHS. The main goal of Bas and Moustafa is to explore the use of machine learning models for RTHS and verify the necessary communication schemes in doing so. Machine learning techniques have been gaining popularity in modeling complex engineering systems across different disciplines. In this study, deep long short-term memory networks in Python are considered for advanced metamodeling for RTHS tests and the idea of embedding the delay compensators within the machine learning model is also explored. The RTHS tests are validated through comparisons with the pure analytical solutions obtained from finite element models.

Most of the above hybrid simulation research works are related to earthquake engineering. As hybrid simulation evolves with more powerful tools and enabling techniques, more and more researchers start the exploration of hybrid simulation in other engineering fields. Moni et al. introduce a new testing 
platform for wind engineering, which combines a numerical simulation and the conventional aeroelastic wind tunnel test through RTHS. The stiffness, damping, and partial mass of a scaled building model are represented as the numerical subsystem, while the rest of the mass, the wind-induced pressure around the model and the wind-structure interaction are represented as the experimental subsystem in a wind tunnel. This RTHS framework is validated and calibrated by a series of preliminary tests and RTHS tests in this study. In Song et al., a RTHS framework for monopile offshore wind turbine (OWT) is proposed to study the structural behavior of OWTs under combined wind-wave loading conditions. To overcome the scaling incompatibilities in OWT scaled model testing, the rotor assembly is replaced by an aerodynamic model simulated in full-scale, representing the numerical subsystem, whereas the experimental subsystem contains a reduced-scale tower structure along with the hydrodynamic loading effects provided by wave tank. A set of sensitivity analyses is conducted to evaluate the feasibility of this RTHS framework and determine possible influence of scaling laws, noises, and delays on its design.

With comprehensive reviews to the past technologies and development of more powerful enabling tools and new applications across broader engineering fields, the authors contributing to this Research Topic not only advance the knowledge of hybrid simulation through their latest research works, but also provide exploratory and inspirational fronts for its future growth and expansion. The collection made through this Research Topic is a timely snapshot of these exciting technical advancements which may propel hybrid simulation into practice, and more importantly, we hope it could highlight the new research avenues for more to explore.

\section{AUTHOR CONTRIBUTIONS}

WS drafted the document. C-MC and VD reviewed and provided revising comments on the document. All authors contributed to the article and approved the submitted version.

\section{ACKNOWLEDGMENTS}

The topic editors would like to thank all the contributors to this Research Topic for submitting their valuable research works for consideration. They would also like to extend their sincere appreciation to the reviewers and editorial team of Frontiers for their help and effort to make this collection possible.

Conflict of Interest: The authors declare that the research was conducted in the absence of any commercial or financial relationships that could be construed as a potential conflict of interest.

Copyright (c) 2020 Song, Chang and Dertimanis. This is an open-access article distributed under the terms of the Creative Commons Attribution License (CC BY). The use, distribution or reproduction in other forums is permitted, provided the original author(s) and the copyright owner(s) are credited and that the original publication in this journal is cited, in accordance with accepted academic practice. No use, distribution or reproduction is permitted which does not comply with these terms. 